\title{
Chicken novel leukocyte immunoglobulin-like receptor subfamilies B1 and B3 are transcriptional regulators of major histocompatibility complex class I genes and signaling pathways
}

\author{
Anh Duc Truong ${ }^{1,2}$, Yeojin Hong', Janggeun Lee', Kyungbaek Lee ${ }^{1}$, Ha Thi Thanh Tran², Hoang Vu Dang ${ }^{2}$, \\ Viet Khong Nguyen ${ }^{2}$, Hyun S. Lillehoj ${ }^{3}$, and Yeong Ho Hong ${ }^{1, *}$
}

\begin{abstract}
* Corresponding Author: Yeong Ho Hong Tel: +82-31-670-3025, Fax: +82-31-671-3025, E-mail: yhong@cau.ac.kr
\end{abstract}

'Department of Animal Science and Technology, Chung-Ang University, Anseong 17546, Korea

2 Department of Biochemistry and Immunology, National Institute of Veterinary Research, 86 Truong Chinh, Dong Da, Hanoi 100000, Vietnam

${ }^{3}$ Animal Biosciences and Biotechnology Laboratory, Agricultural Research Services, United States

Department of Agriculture, Beltsville, MD 20705, USA

ORCID

Anh Duc Truong

https://orcid.org/0000-0002-2472-8165 Yeojin Hong

https://orcid.org/0000-0001-8071-8183

Janggeun Lee

https://orcid.org/0000-0002-8552-9581

Kyungbaek Lee

https://orcid.org/0000-0002-7887-2437 Ha Thi Thanh Tran

https://orcid.org/0000-0001-7342-8815 Hoang Vu Dang

https://orcid.org/0000-0003-0006-7902

Viet Khong Nguyen

https://orcid.org/0000-0002-8383-0223

Hyun S. Lillehoj

https://orcid.org/0000-0001-7755-6216

Yeong Ho Hong

https://orcid.org/0000-0002-4510-7851

Submitted Jul 27, 2018; Revised Sept 20, 2018; Accepted Oct 4, 2018
Objective: The inhibitory leukocyte immunoglobulin-like receptors (LILRBs) play an important role in innate immunity. The present study represents the first description of the cloning and structural and functional analysis of LILRB1 and LILRB3 isolated from two genetically disparate chicken lines.

Methods: Chicken LILRB1-3 genes were identified by bioinformatics approach. Expression studies were performed by transfection, quantitative polymerase chain reaction. Signal transduction was analyzed by western blots, immunoprecipitation and flow cytometric. Cytokine levels were determined by enzyme-linked immunosorbent assay.

Results: Amino acid homology and phylogenetic analyses showed that the homologies of LILRB1 and LILRB3 in the chicken line 6.3 to those proteins in the chicken line 7.2 ranged between $97 \%-99 \%$, while homologies between chicken and mammal proteins ranged between $13 \%-19 \%$, and $13 \%-69 \%$, respectively. Our findings indicate that LILRB1 and LILRB3 subdivided into two groups based on the immunoreceptor tyrosine-based inhibitory motifs (ITIM) present in the transmembrane domain. Chicken line 6.3 has two ITIM motifs of the sequence LxYxxL and SxYxxV while line 7.2 has two ITIM motifs of the sequences LxYxxL and LxYxxV. These motifs bind to SHP-2 (protein tyrosine phosphatase, non-receptor type 11) that plays a regulatory role in immune functions. Moreover, our data indicate that LILRB1 and LILRB3 associated with and activated major histocompatibility complex (MHC) class I and $\beta 2$-microglobulin and induced the expression of transporters associated with antigen processing, which are essential for MHC class I antigen presentation. This suggests that LILRB1 and LILRB3 are transcriptional regulators, modulating the expression of components in the MHC class I pathway and thereby regulating immune responses. Furthermore, LILRB1 and LILRB3 activated Janus kinase2/tyrosine kinase 2 (JAK2/TYK2); signal transducer and activator of transcription $1 / 3$ (STAT1/3), and suppressor of cytokine signaling 1 genes expressed in Macrophage (HD11) cells, which induced Th1, Th2, and Th17 cytokines.

Conclusion: These data indicate that LILRB1 and LILRB3 are innate immune receptors associated with SHP-2, MHC class I, $\beta 2$-microglobulin, and they activate the Janus kinase/signal transducer and activator of transcription signaling pathway. Thus, our study provides novel insights into the regulation of immunity and immunopathology.

Keywords: Chicken; Leukocyte Immunoglobulin-like Receptor 1 (LILRB1); LILRB3; Major Histocompatibility Complex (MHC) Class I; Cytokines

\section{INTRODUCTION}

In mammals, leukocyte immunoglobulin-like receptors (LILRs) include 11 functional genes, which are classified as activating (LILRA1, 2, 4-6), inhibitory (LILRB1-5), and soluble (LILRA3). They are highly homologous in their extracellular regions but differ in their intracellular 
regions [1,2]. The leukocyte immunoglobulin (Ig)-like receptor subfamily B (LILRB) of humans was first described and cloned in 1997 [3] and acts as an important group of immunoreceptor tyrosine-based inhibitory motifs [ITIMs: I/V/L/S) $\mathrm{XYXX}(\mathrm{L} / \mathrm{V})$ ] containing receptor in their cytoplasmic tail. It belongs to a group of type I transmembrane glycoproteins with extracellular Ig-like domains that bind ligands and intracellular ITIMs [4,5]. The structure of LILRB groups consists of a signal peptide, two or four Ig-like domains, transmembrane domain and a long cytoplasmic tail with ITIMs $[1,6]$. Moreover, the LILRB groups are classified into group 1 (LILRB1 and LILRB2) and group 2 (LILRB3, LILRB4, and LILRB5). They consist of members that interact with the human leukocyte antigen (HLA) class I molecules and non-HLA ligands and are well-characterized and have been increasingly identified in the recent years $[1,2,6]$.

These LILRB groups are expressed on various cell types such as macrophages, T cells, B cells, NK cells, and dendritic cells although the expression patterns are different between them $[1,2,6]$. ITIMs are activated by various phosphatases such as protein tyrosine phosphatase, non-receptor type 6 (PTPN6 or SHP-1), SHP-2 (PTPN11), or Src homology 2 domain-containing inositol phosphatase (SHIP) [1]. These phosphatases are capable of dephosphorylating multiple signaling-related molecules such as extracellular-regulated kinase (ERK), Janus kinase/ signal transducer and activator of transcription (JAK/STAT), mitogen-activated protein kinase (MAPK) or nuclear factor kappa B subunit signaling pathway [7]. LILRB groups have been shown to control the activity of toll-like receptors (TLR) [8], antigen-presenting and cytokine production, thus demonstrating that the LILRB groups play an important role in the regulation of innate immune responses [8]. Moreover, the gene expression and polymorphisms of LILRBs have been reported to be associated with autoimmune and infectious diseases such as those caused by Salmonella [8], cytomegalovirus [9], and Mycobacterium tuberculosis [10], as well as rheumatoid arthritis [11], Alzheimer's model [12], psoriatic arthritis [13], HIV/ AIDS [14], and cancer [6]. Recently, some members of the Ig superfamily that may be involved in immune responses such as triggering receptor expressed on myeloid cells, cluster of differentiation 300 , signal regulatory protein and chicken Ig-like receptors (CHIR)-A, CHIR-B, and CHIR-AB homologs were identified in chicken using bioinformatics approaches [15]. The studies of the chicken leukocyte immunoglobulin receptor (LIR) indicated that LIR genes are shown highly polymorphic locus harboring a variable number of CHIR genes and also play an important role in avian influenza infection in chicken [15]. However, pertinent information is lacking in chicken; for example, no data are available on the primary structure of LILRB1 and LILRB3, and the functions of those have not yet been investigated.

In this paper, therefore, we have identified novel LILRB1 and LILRB3 genes and described the expression and functional analysis of the variants of LILRB1 and LILRB3 genes in chicken, namely LILRB1R, -B1S, -B3R, and -B3S, which are isolated from two genetically disparate chicken lines in macrophage (HD11) cell lines. Our findings strongly suggest that the LILRB1 and LILRB3 associated with major histocompatibility complex (MHC) class I and non-classical $\beta 2$-microglobulin ( $\beta 2 \mathrm{~m})$ and controlled the constitutive signaling mediated by SHP-2 that regulates the JAK/STAT signaling pathway. We also described that LILRB1 and LILRB3 were expressed on HD11 cells and they modulated cytokines production.

\section{MATERIALS AND METHODS}

\section{Bioinformatic analysis and identification of chicken LILRB 1 and $B 3$ genes}

Our previous study $[16,17]$ reported that more than 15,000 novel transcripts are expressed in the chicken lines 6.3 (R) and 7.2 (S), which associated with Marek's disease (MD) resistance and shared the same MHC haplotype. These sequences were then used to search all the current chicken genome assemblies through the Gallus_gallus-5.0 reference Annotation Release 103 nucleotide BLAST program (https://blast.ncbi.nlm.nih.gov/ Blast.cgi). The chromosomal locations were clarified using the BLAST-Like Alignment Tool (BLAT) (http://asia.ensembl. org/Gallus_gallus/Info/Index) (Supplementary Table S1). The genomic DNA identified as encoding potential chicken LILRB1 and LILRB3 genes was then analyzed using GeneScan [18] to predict the coding DNA sequence (CDS) and protein domains. To confirm the sequences of two genetically disparate chicken lines, primers were designed using the Lasergene software (DNASTAR Inc. Madison, WI, USA) and synthesized by Genotech Co. Ltd. (Daejeon, Korea) (Supplementary Table S2). The polymerase chain reaction (PCR) products from 10 individual samples of chicken line 6.3 and line 7.2 were purified using the QIAQuick gel Extraction Kit (QIAgen, Hilden, Germany), and sub-cloned into the pCR2.1-TOPO vector (Invitrogen, Carlsbad, CA, USA), followed by transformation into Escherichia coli (E. coli) TOP10 (Invitrogen, USA) highefficiency competent cells. Through blue-white screening, the positive clones were selected and sequenced by Genotech (Korea). Protein identification was conducted using the Expert Protein Analysis System (ExPASy; http://www.expasy. org/tools/) and the multiple sequence alignment was analyzed using the Lasergene software (DNASTAR Inc., USA). To draw a phylogenetic tree of the amino acid sequences of LILRB1 and LILRB3 groups, the neighbor-joining method with a bootstrap value of 1,000 in the MEGA6 program (https://www. megasoftware.net/) was used. Signal peptides were predicted by SignalP 4.1 Server v.4.1 (http://www.cbs.dtu.dk/services/ SignalP/) and glycosylation motifs were predicted using NetOGlyc 4.0 Server (www.cbs.dtu.dk/services/NetOGlyc/). The 
Ig domains, transmembrane domain, and the cytoplasmic regions were predicted by InterPro v.56.0 (https://www.ebi.ac.uk/ interpro/). The ITIMs were mapped to each peptide sequence using the established V/L/S/NxYxxL/V ITIM motif [4,5]. Fulllength CDS of LILRB1 and LILRB3 genes were cloned in pCR2.1 (Invitrogen, USA) and excised from pCR2.1 using NotI/Xbal (Bioneer Corp, Daejeon, Korea), and ligated into the eukaryotic expression vector, pcDNA3-eGFP (Addgene, Cambridge, MA, USA), and then, followed by the transformation of E. coli BL21 (Invitrogen, USA). The positive clones were sequenced, and the structure and the ligand-binding site of the protein were determined by molecular replacement using the program RaptorX web server (http://raptorx.uchicago.edu/). To identify the function, the ligands of LILRB1 and LILRB3 genes were mapped and searched using the RCSB protein data bank (http://www.rcsb.org).

\section{Transfection into the macrophage (HD11) cell line}

The chicken macrophage (HD11) cells [19] were transiently transfected with a pcDNA3-eGFP vector containing either the LILRB1R-, LILRB1S-, LILRB3R-, or LILRB3S-encoding sequence and also mock control with empty pcDNA3-eGFP vector using Lipofectamine 3000 transfection reagent (Invitrogen, USA), as per the manufacturer's recommended protocols. A total of $1.0 \times 106$ cells per well in six-well plate (Thermo Scientific, Waltham, MA, USA) were transfected with $4.0 \mu \mathrm{g}$ of plasmid using the Lipofectamine 3000 reagent and the transfected cells were harvested after $48 \mathrm{~h}$ for further analysis.

\section{Reagents}

Mouse monoclonal anti-GFPuv/eGFP antibody was purchased from R\&D Systems (Minneapolis, MN, USA). Mouse antichicken MHC class I-PE and mouse anti-chicken $\beta 2 \mathrm{~m}-\mathrm{PE}$ antibodies were purchased from Southern Biotech (Birmingham, AL, USA). Rabbit anti-chicken p-STAT1 $\left(\mathrm{Ser}^{727}\right)$, antichicken p-STAT3 $\left(\operatorname{Ser}^{727}\right)$, and anti-chicken p-JAK2 $\left(\operatorname{Tyr}^{100} 7 /\right.$ $\mathrm{Tyr}^{1008}$ ) were purchased from Santa Cruz Biotech (Dallas, TX, USA). Rabbit anti-chicken suppressor of cytokine signaling 1 (SOCS1), anti-chicken STAT1, anti-chicken STAT3 antibodies, horseradish peroxidase (HRP)-linked anti-rabbit secondary antibodies, and protein G-sepharose beads were purchased from Sigma-Aldrich (St. Louis, MO, USA). Rabbit anti-chicken p-SHP2 $\left(\mathrm{Tyr}^{542}\right)$, rabbit anti-chicken JAK2, and rabbit antichicken TYK2 antibodies were purchased from Biorbyt (San Francisco, CA, USA). Rabbit anti-chicken glyceraldehyde-3phosphate dehydrogenase (GAPDH) antibody was purchased from Abcam (Cambridge, MA, USA). Anti-His (C-Term)-HRP and Alexa Fluor 488 goat anti-rabbit IgG $(\mathrm{H}+\mathrm{L})$ secondary antibodies were purchased from Invitrogen (USA). Mouse monoclonal anti-chicken interferon (IFN)- $\gamma$ antibody, mouse monoclonal anti-chicken interleukin (IL)-17A antibody, mouse monoclonal anti-chicken IL-12p40 antibody and recombi- nant versions of these proteins (kindly provided by Dr. Hyun S. Lillehoj, USDA), EZ-Link Sulfo-NHS-LC-Biotin, Goat antimouse IgG secondary antibody linked HRP conjugate, and HRP-conjugated Streptavidin were purchased from Thermo Scientific (USA).

\section{Flow cytometry analysis}

The cells were incubated in a flow cytometer buffers ( $10 \%$ fetal calf serum), 15 mM 4-(2-hydroxyethyl)-1-piperazineethanesulfonic acid, and $2 \mathrm{mM}$ ethylenediaminetetraacetic acid [EDTA] in phosphate buffered saline [PBS]) and primary antibodies were added to $1.0 \times 10^{6}$ cells and then, kept for $30 \mathrm{~min}$ on ice. To evaluate the LILRB1- and LILRB3-eGFP binding with MHC class I and $\beta 2 \mathrm{~m}$, anti-MHC class I-PE and anti- $\beta 2 \mathrm{~m}-\mathrm{PE}$ antibodies were incubated with the cells as described above, followed by a single wash with the flow cytometer buffers. For assessing LILRB1 - and LILRB3-eGFP constitutive signaling mediated by SHP-2, anti-SHP-2 antibody was added and incubated for $30 \mathrm{~min}$ on ice, followed by a single wash with the flow cytometer buffers before adding Alexa Fluor 488-conjugated antirabbit secondary antibody (Invitrogen, USA). The control groups were incubated with goat anti-chicken IgG-PE or rabbit IgG only (data not show). Flow cytometry analysis was performed with BD FACS Aria II cell analyzer (BD Biosciences, San Jose, CA, USA). Data were acquired with BD FACS Diva Version 6.1.3 and analyzed using FlowJo 7.6.1. Forward and side-scatter gating removed contaminants such as cell debris.

\section{Immunoprecipitation and western blotting}

Cells were washed twice with ice-cold PBS, harvested in icecold RIPA buffer (50 mM Tris- $\mathrm{HCl}$ pH 7.5, $150 \mathrm{mM} \mathrm{NaCl}, 2$ mM EDTA, $100 \mathrm{mM}$ sodium fluoride, $0.1 \%$ [w/v] sodium dodecyl sulfate [SDS], $0.5 \%[\mathrm{w} / \mathrm{v}$ ] sodium deoxycholate, $1 \%$ Triton $\mathrm{X}-100,10 \mathrm{mM}$ sodium pyrophosphate, and $10 \mathrm{mM}$ sodium orthovanadate) containing complete EDTA-free protease inhibitor cocktail (Thermo Scientific, USA), then gently lysed for $30 \mathrm{~min}$ at $4^{\circ} \mathrm{C}$, and centrifuged at 13,000 g for $15 \mathrm{~min}$ at $4^{\circ} \mathrm{C}$. The concentration of protein was evaluated using the Coomassie protein assay kit (Thermo Scientific, USA) in microplates as per the manufacturer's instructions. The protein $(100 \mu \mathrm{g})$ was incubated with the anti-eGFP $\mathrm{mAb}$ or antiGAPDH antibody at $4^{\circ} \mathrm{C}$ overnight and then added to $50 \mu \mathrm{L}$ of protein G-Sepharose (Invitrogen, USA) for $2 \mathrm{~h}$. The immunoprecipitates were then washed three times in RIPA buffer and solubilized in $2 \times$ SDS-polyacrylamide gel electrophoresis (PAGE) sample buffer. Samples were electrophoresed on Trisglycine SDS-PAGE gels, and the proteins were transferred on to polyvinylidene fluoride membranes (GE Healthcare, Rydalmere, Australia). Membranes were blocked with 5\% non-fat milk (Thermo Scientific, USA) or 3\% bovine serum albumin (BSA) (Sigma-Aldrich, USA) in PBS pH 7.4 containing 0.05\% Tween 20 (PBST) for $2 \mathrm{~h}$ at room temperature (RT, $25^{\circ} \mathrm{C}$ ), 
washed with PBST, and incubated with anti-MHC class I, anti- $\beta 2 \mathrm{~m}$ or anti-SHP-2 antibody or signaling antibodies overnight at $4^{\circ} \mathrm{C}$ and the relevant secondary antibody in $2 \%$ non-fat milk or $0.5 \%$ BSA in PBST for $2 \mathrm{~h}$ at RT. Finally, the membranes were developed using Western Lightning ECL Plus (Thermo Scientific, USA) and Hyperfilm (GE Healthcare, Australia).

\section{Enzyme-linked immunosorbent assay}

We coated a 96-well plate (Nunc MaxiSorp, Nunc, Wiesbaden, Germany) with dilutions (1:500) of monoclonal anti-IFN- $\gamma$, anti-IL-12p40, or anti-IL-17A antibodies for $7 \mathrm{~d}$ at $4^{\circ} \mathrm{C}$ as previously described [20]. Next, the plates were blocked with $5 \%$ non-fat milk for $2 \mathrm{~h}$ at $25^{\circ} \mathrm{C}$, the plates were incubated overnight at $4^{\circ} \mathrm{C}$ with the cell supernatant, or different dilutions of the recombinant IFN- $\gamma$, IL-17A, and IL-12p40. Following incubation with a biotinylated monoclonal IFN- $\gamma$, IL-17A, or IL-12p40 antibody, HRP-conjugated Streptavidin was added. The plates were washed three times with PBST at each step. Then, 3,3',5,5'-tetramethylbenzidine (Thermo Scientific, USA) was used as the chemiluminescent substrate and the luminescence was measured using a Hybrid Microplate Reader (Epoch, BioTek Instruments, Inc, Winooski, VT, USA).

\section{Quantitative real-time polymerase chain reaction}

After $48 \mathrm{~h}$ of transfection with a pcDNA3-eGFP vector containing either the LILRB1R, -B1S, -B3R, or -B3S encoding sequence and a mock control, RNA was extracted using the TRIzol kit (Invitrogen, USA) as per manufacturer's instructions. Next, $2 \mu \mathrm{g}$ total RNA was reverse transcribed to cDNA by using Maxima First Strand cDNA Synthesis Kit (Thermo Scientific, USA), according to manufacturer's protocols. Quantitative PCR (qPCR) was performed using Light Cycler 96 Real-time PCR System (Roche Diagnostics, Indianapolis, IN, USA) in a $20-\mu \mathrm{L}$ reaction mixture containing $1 \mu \mathrm{L} c \mathrm{cNA}, 10 \mu \mathrm{L}$ $2 \times$ FastStart Universal SYBR Green Master Mix (Roche Diagnostics, USA), and 10 pmol each of forward and reverse primers of genes (Supplementary Table S2). The primers were designed using the Lasergene software (DNASTAR Inc., USA) and were synthesized by Genotech Co. Ltd. (Korea). Thermal conditions for performing $\mathrm{qPCR}$ are as follows: initial incubation at $95^{\circ} \mathrm{C}$ for $5 \mathrm{~min} ; 40$ cycles of denaturation at $95^{\circ} \mathrm{C}$ for $30 \mathrm{~s}$, annealing at $50^{\circ} \mathrm{C}$ to $55^{\circ} \mathrm{C}$ for $30 \mathrm{~s}$, and extension at $72^{\circ} \mathrm{C}$ for $30 \mathrm{~s}$; and termination by final incubation at dissociation temperatures $95^{\circ} \mathrm{C}(10 \mathrm{~s}), 65^{\circ} \mathrm{C}(60 \mathrm{~s}), 97^{\circ} \mathrm{C}(1 \mathrm{~s})$, and $37^{\circ} \mathrm{C}$ (30 s). Genes expression were quantified after normalization with the chicken GAPDH gene and were calculated using the $2^{-\Delta \Delta C t}$ method.

\section{Bioactivity assays}

To determine the cytotoxicity of LILRB1R, -B1S, -B3R, and -B3S linked eGFP vector and empty vector transfected into
HD11 cell line after $48 \mathrm{~h}$ transfection, cell proliferation and NO production assay was performed in 96-well plates according to well-established protocols [20]. To measure the nitrite content, $100 \mu \mathrm{L}$ of the culture medium was incubated with $100 \mu \mathrm{L}$ of Griess reagent (Sigma-Aldrich, USA) at RT for 10 min. Then, the absorbance was measured at $540 \mathrm{~nm}$ using a microplate reader as described [20]. The nitrite content was calculated based on a standard curve constructed with $\mathrm{NaNO}_{2}$. Cell proliferation was determined with the cell counting Kit8 (CCK-8) assay according to the manufacturer's protocol as described [20] (Dojindo Molecular Technologies, Inc., Mashikimachi, Kumamoto, Japan). Lipofectamine 3000 transfection reagent and culture medium were used as controls.

\section{Statistical analysis}

Data are represented as the mean \pm standard error of the mean of three independent experiments for each group $(n=3)$ and were analyzed with the SAS 9.4 statistical program (SAS Institute Inc., 100 SAS Campus Drive Cary, NC, USA). Comparison between the experimental groups was carried out using Duncan's multiple comparison method. Differences were considered significant when $\mathrm{p}<0.05$.

\section{RESULTS}

Identification of potential chicken LILRB1 and LILRB3 Based on the RNA-Seq results from two genetically disparate Necrotic Enteritis (NE)-afflicted chicken lines [16,17], we identified 11 open reading frames (ORFs) in our library as containing potential chicken LILRB1 and LILRB3 genes. Each of these ORFs was then analyzed by GeneScan and BLAT to obtain the predicted CDS and the corresponding protein sequence, which were then aligned to domains using protein BLAST to identify the Ig domains. All 11 ORFs were located on chromosome Un-random from 3,559,608 to $63,671,960$ positions. Of the 11 ORF sequences, we considered 4 ORFs sequence groups, which contain Ig domains and showed different sequences between the two chicken lines. Then, we evaluated the expression levels of LILRB1R, $-B 1 S,-B 3 R$, and - B3S transcripts in both the NE-induced chicken lines, and the results showed that the expression of LILRB1 and LILRB3 transcripts in the chicken line 6.3 was higher than that of line 7.2 although the expression of these transcripts in both the NE-induced lines was higher than that of the control (Supplementary Table S1). Next, the novel mRNA transcripts of $L I L R B 1 R,-B 1 S,-B 3 R$, and -B3S were amplified by RT-PCR, cloned, and sequenced for 10 individual samples from each chicken line. Nucleotide and amino acid sequence analyses showed that the identity of LILRB1 or LILRB3 in 10 samples of each chicken line was 100\% (data not shown). Analysis of the LILRB1R, $-B 1 S,-B 3 R$, and -B3S nucleotide sequences showed a $30 \%$ to $38 \%$ similarity with mammals such as human, 
mouse, monkey, chimpanzee, and pig (data not shown). Multiple alignments of each predicted LILRB1 and LILRB3 protein on line 6.3 to those proteins on line 7.2 showed that the amino acids had an identity and similarity range of $97 \%$ to $99 \%$ (Table 1). Comparison of amino acid identities and similarities between LILRB1 and LILRB3 in chicken to mammals ranked between $13 \%$ to $19 \%$ and $13 \%$ to $69 \%$, respectively (Table 1). Based on amino acid similarities, phylogenetic analysis placed the novel receptors together with the members of the mammalian LILRB1 and LILRB3 genes that were themselves clustered using the neighbor-joining bootstrap consensus trees inferred from 1,000 replicates (Figure 1A). The results indicated that these 4 receptors are closely associated with the LILRB1 and LILRB3 genes of other species. Specifically, it can be concluded that the LILRB1R, -B3R, and LILRB1S, -B3S amino acids isolated in chicken line 6.3 and line 7.2, respectively, are homologous based on the high degree of sequence similarity (Figure 1A). From these results, the newly identified transcripts from the two genetically disparate chicken lines can be classified as chicken LILRB1R, -B1S, -B3R, and -B3S.

\section{Structure and potential ligand binding of novel LILRB1/3 genes}

Overall, the secondary structure of chicken LILRB1 and LILRB3 proteins included the signal peptide, Ig domains, transmembrane, cytoplasmic domain, and two ITIM motifs (Figure 1B). Based on the Ig domain structure, we also divided these genes into 2 subgroups as group 1 (LILRB1R and LILRB1S) and group 2 (LILRB3R and LILRB3S). Group 1 contains 2 Ig domains $\left(\mathrm{W}^{54}-\mathrm{Y}^{147}\right.$ and $\left.\mathrm{S}^{150}-\mathrm{A} / \mathrm{V}^{231}\right)$ and two $\mathrm{N}$-glycosylations $\left(\mathrm{N}^{137}\right.$ and $\left.\mathrm{N}^{207}\right)$ whereas group 2 contains one Ig domain $\left(\mathrm{L}^{23}\right.$ $\mathrm{D}^{116}$ ) and three $\mathrm{N}$-glycosylations $\left(\mathrm{N}^{138}, \mathrm{~N}^{148}\right.$, and $\mathrm{N}^{268}$ ) (Figure 1B). Based on the presence or absence of ITIMs, the signaling domains found in the chicken LILRB1 and LILRB3 genes are different than those in humans. The human LILRB1 and LILRB3 genes have 4 ITIMs present in the LILRB1 and LILRB3 receptors: NxYxxV, VxYxxV, VxYxxL, and SxYxxL, whereas the chicken LILRB1 and LILRB3 genes contain 2 ITIMs, one of which, $\mathrm{LxYxxL}$ is common to all receptors (Figure 1C). It is suggested that human LILRB1 and LILRB3 genes might differ in their capacity of their ligand binds and regulate immune responses compared to chicken LILRB1 and LILRB3 genes. On the other hand, the second ITIMs motifs were different between LILRB1 and LILRB3 genes isolated from the two genetically disparate chicken lines. The SxYxxV motif appears in the resistant chicken lines (LILRB1R, -B3R) while LxYxxV in the susceptible lines (LILRB1S, -B3S) (Figure 1C). This suggests that the LILRB1 and LILRB3 genes contribute differently to cellular regulation and innate immunity in resistant and susceptible chicken lines. Moreover, in humans, it is reported that the ITIMs motif also determined the binding of the cytosolic tyrosine kinases SHP-1 and SHP-2 [4]. Thus, the results suggest that the ITIMs motif in the two genetically different chicken lines may be associated with tyrosine kinases and regulation of signaling pathways. Consequently, we concluded that our newly identified transcripts are significantly different in the two genetically disparate chicken lines. Therefore, it is suggested that chicken LILRB1 and LILRB3 proteins are similar to those in humans in structure and function and may differ only in their ligand binding sites.

Identification of ligands for LILRB1 and LILRB3 genes is a key step in clarifying the biology of LILRB family genes and understanding the function of these genes. The ligands for LILRB3 is not clear in humans. Moreover, LILRB1 has multiple ligands that bind with MHC class I [2]. Using the RaptorX web server program, we screened candidate ligands of LILRB1R,

Table 1. Similarity (upper) and identity (below) of LILRB1 and LILRB3 genes from two genetically disparate chicken lines and other species

\begin{tabular}{|c|c|c|c|c|c|c|c|c|c|c|c|c|c|c|c|}
\hline \multirow{2}{*}{ Species } & \multirow{2}{*}{ Genes } & B1R & B1S & B1 & B1 & B1 & B1 & B3R & B3S & \multirow{2}{*}{$\frac{\text { B3 }}{\text { Human }}$} & \multirow{2}{*}{$\frac{\text { B3 }}{\text { Chimp }}$} & \multirow{2}{*}{$\begin{array}{c}\text { B3 } \\
\text { Mouse }\end{array}$} & \multirow{2}{*}{ B3 } & \multirow{2}{*}{$\begin{array}{c}\text { B3 } \\
\text { Pig }\end{array}$} & \multirow{2}{*}{ - GenBank Acc. } \\
\hline & & \multicolumn{2}{|c|}{ Chicken } & Human & Chimp & Mouse & Pig & Chic & & & & & & & \\
\hline hicken & & - & 99.10 & & & & & 79.97 & & & & & & & \\
\hline hicken & 15 & 97.53 & - & 38.24 & 35.09 & & 67.37 & & & & & & & & \\
\hline uman & & & & - & & & & & & & & & & & \\
\hline himpa & B1 & & & & - & & & & & & & & & & \\
\hline louse & B1 & & & & & - & 43.86 & & & & & & & & \\
\hline ig & B1 & & & & & & - & 65.69 & & & & & & & \\
\hline nicken & & & & & & & & - & & & & & & & \\
\hline hicken & & & & & & & & & - & & & & & & \\
\hline uman & B3 & & & & & & & & & & 93.70 & & & & \\
\hline himpan & B3 & & 16.35 & & & & & & & & - & 49.26 & & 41.61 & 09055 \\
\hline Mouse & B3 & & & & & & & & & & & - & 46.56 & & \\
\hline & B3 & 16.61 & & & & & & & & & & & - & 35.20 & \\
\hline Pig & B3 & 16 & 16.66 & 26.76 & 27.69 & 25.23 & 18.32 & 15.38 & 15.69 & 25.84 & 25.23 & 24 & 25.53 & - & XP_013854226 \\
\hline
\end{tabular}

LILRB, leukocyte immunoglobulin-like receptors.

The nucleotide sequence of LILRB1 and LILRB3 genes were identified from RNA-Seq data in spleen and intestinal mucosa layer of two genetically disparate chicken lines afflicted with necrotic enteritis. 
A

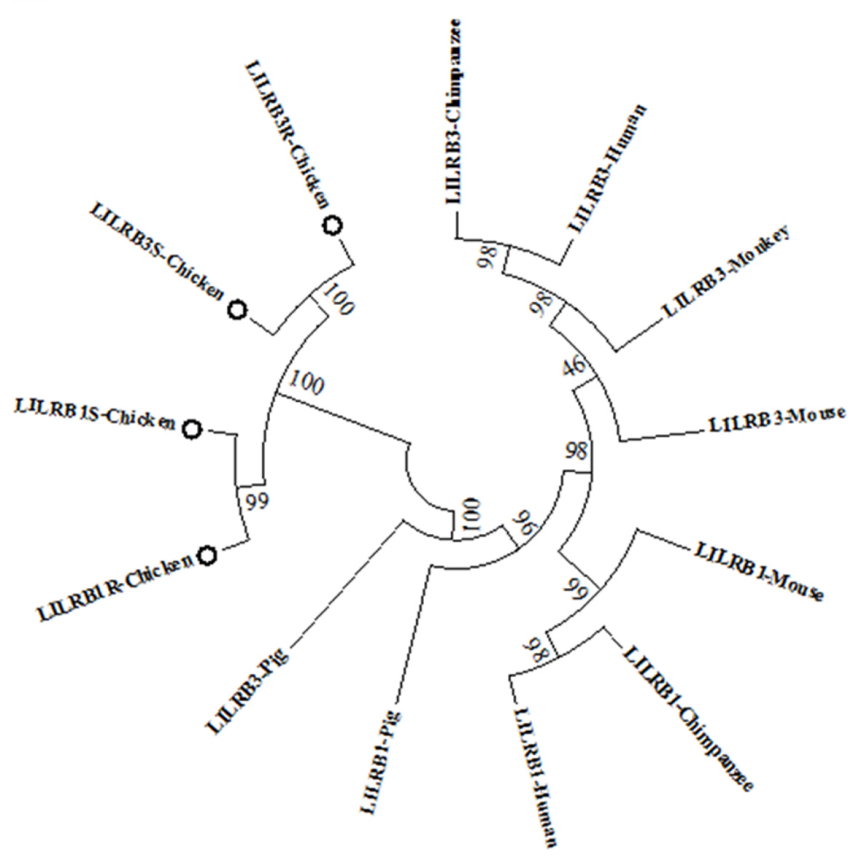

B LILRB1R

LILRB1S

LILRB3S

LILRB3R

LILRB1R

LILRB1S

LILRB3S

LILRB3R

LILRB1R

LILRB1S

LILRB3S

LILRB3R

LILRB1R
LILRB1S

LILRB3S

LILRB3R

LILRB1R

LILRB1S

LILRB3S

LILRB3R

LILRB1R

LILRB1S

LILRB3S

LILRB3R

C
MAPMAVALILGWWLVAASRAQQLPRPSLSLHPSQRVSLGDTVTLRCHLPQPAAWVELYQ MAPMAVALILGWWLVAASRAOOLPRPSLSLHPSQGVSLGDTVTLRCHLPRPAA-WVILYQ MAPMVVAHILSWWLVAASRAQ $Q$ LSRPFLSLHPS $Q$ GVSLGDTVTLRCHLR LAR VWL MAPMVVAH ILSWWLVAASRAQQLPRPFLSLHPSQ QVVSLGDLVTLRCHLPRLAAWVWLYQ

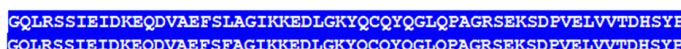
¿LLRSIEIDKEQQDVAEFSFAGIKKEDLGKYQCQYQGLQPAGRSEKSDPVELVVIDHSY GTLRENKYKDKELDAAEFS SMTTSWEDAGTYRCQYHVSD PLGTSEKSDPVELVLTDGQV GTLRENKYKDKELDAAEFS SMTTSWEDAGMYRCQYHVSDPLGTSEKSDPVEIVLTDGQVP

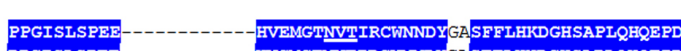
PRGISLSPEE----------HVGMGTNVTIRCWNNDY GA SFFLHKDGHSAPLOHOEP SIPLPAHQLOLPGTQHFTNLSIHVGMGSNVTIQCWNWDYGSTFLLHQGLGTHPLSPVDSSIPLPAHQLQLPGTQHFT NLSIHVGMGFNVTIQCWNWDYGSTFLLHQGLGTQPLSSVDS

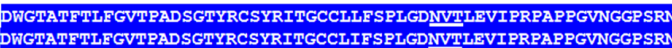
DVVDVTPTPATPVSSETPEVGR --MVVDVTPTPATPVSSETPFVGPQCQPWVELSRHP--CPVMGTPKHSEPTGAKRRSYVI $* * *: \quad: \quad . \quad:{ }^{*}$

†Cytoplasmic domain

LVATVAGGCVAAHCIHIRLTASLLLVARRQMLSDLRPGATSRGPEAVQFQVSPGDSEG WVAVAGGCVAAHCIHICLTASLLLVAQRRQMLSDLRPGATSRGPEAVQFQVSPGDSEG LVVVVIVCSALLALSSGLYFYFNGCSLWKRRNES---AAWYHPTVPLCLQVPPADNEG LVVVVVNVCSALLALSSGLYFYANGCSLWKRRNES---AAWYHPTVPLCLQVPPADNEG $: \quad:: 0^{*}: . .::^{* *} .^{*} .^{*} .^{*}$

TTYAQIQAVTPSTHPRGSSTTPEPPSI YAEVGTRGPR TTYALIQAVTPSTHPRGSSTTPE PPLIYAEVGTRGPR LTYTEIOAVTPSFCR PGPSAVPOAPSI YAQVGFGEPH LTYTEIQAVTPSFCRPGPSAVPQAPLI YAQVGFGEPH
$* * *: * * * * * * * * * * * * * * * * *: * * *$

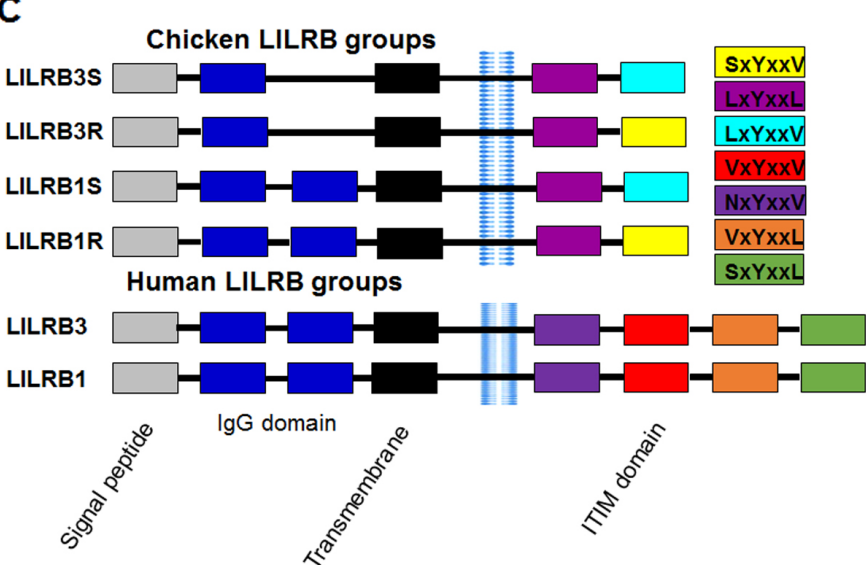

Figure 1. Genetic analysis of LILRB1 and LILRB3 genes identified from two genetically disparate chicken lines. (A) Phylogenetic tree indicating the relationship between chicken LILRB1 and LILRB3 amino acid sequences and those of other vertebrates; (B) Multiple alignment of the predicted LILRB1 and LILRB3 genes from two genetically disparate chicken lines was performed using the ClustalX program. Shown is the result of the amino acid sequence alignment of chicken LILRB1 and LILRB3 genes together with human LILRB1 and LILRB3 genes. Signal peptide, Ig domain, transmembrane, and cytoplasmic domain are indicated in dark, blue, black, and no color, respectively. The $\mathrm{N}$-linked glycosylation sites are underlined; (C) Secondary structures of chicken LILRB1 and LILRB3 genes compared to those of the known human LILRB1 and LILRB3 genes. $L I L R B$, leukocyte immunoglobulin-like receptors.

-B1S, -B3R, and -B3S, and identified their function. The ligand of LILRB1 and LILRB3 genes were mapped and searched in the RCSB protein data bank and also in previously reported literature. The results showed that LILRB1 and LILRB3 genes have at least two ligands each and most of the ligand candidates for LILRB1 and LILRB3 genes bound MHC-class I and $\beta 2 \mathrm{~m}$ antigen (Table 2, Supplementary Figure S2). Comparison of the LILRB1 and LILRB3 genes between the two genetically disparate chicken lines showed that both genes had different ligand-binding residues. Therefore, this suggests that LILRB1 and LILRB3 genes may be binding MHC class I, and $\beta 2 \mathrm{~m}$ differently in the two genetically disparate chicken lines and the binding might also differ between LILRB1 and LILRB3 genes.
MHC-class I and $\beta 2$-microglobulin heavy chain binds to LILRB1 and LILRB3

To confirm the efficiency of eGFP-linked with either LILRB1R, -B1S, -B3R, and -B3S and mock control (empty eGFP vector), these constructs were transfected into the HD11 cell line. First, we determined the transfection efficiency in LILRB1 or LILRB3transfected HD11 cell line by fluorescent microscopy and fluorescence-activated cell sorting (FACS) analysis (Supplementary Figure S2A). The transfection efficiency was $>90 \%$ as determined by FACS analysis (Supplementary Figure S2A). Finally, the expressed LILRB1 and LILRB3 proteins were determined by western blotting. As shown in Supplementary Figure S2B, a single band was detected by anti-eGFP $\mathrm{mAb}$ in the eGFP-linked LILRB1 lane and in the LILRB3-transfected 
Table 2. Candidate binding ligands of LILRB1 and LILRB3 genes in two genetically disparate chicken lines

\begin{tabular}{|c|c|c|c|c|c|c|}
\hline Name & Ligand & Binding residues & Domain & p-value & Binding with & References \\
\hline \multirow[t]{2}{*}{ LILRB1R } & ZN & $A^{51} Q^{52} G^{128} P^{131} G^{133} S^{135}$ & $1-151$ & $3.12 \mathrm{E}-07$ & MHC class I & [30] \\
\hline & EDO & $H^{185} K^{186} D^{187} T^{218} Y^{219} R^{220}$ & $152-355$ & $6.25 \mathrm{E}-06$ & MHC class I, $\beta 2 \mathrm{~m}$ & {$[31,32]$} \\
\hline \multirow[t]{2}{*}{ LILRB1S } & ZN & $Q^{52} G^{128} L^{129} Q^{130} P^{131} A^{132}$ & $1-151$ & 5.10E-07 & MHC class I & {$[30]$} \\
\hline & EDO & $H^{185} K^{186} D^{187} T^{218} Y^{219} R^{220}$ & $152-355$ & $7.69 \mathrm{E}-06$ & MHC class I, $\beta 2 \mathrm{~m}$ & {$[31,32]$} \\
\hline \multirow[t]{3}{*}{ LILRB3R } & EDO & $L^{30} P^{32} S^{33} Q^{34} E^{111} L^{112} V^{113}$ & $1-120$ & $1.14 \mathrm{E}-07$ & MHC class I, $\beta 2 m$ & {$[31,32]$} \\
\hline & NAD & $L^{164} G^{200} Q^{202} E^{208} L^{209} S^{210} R^{211} P^{213}$ & $121-247$ & $2.03 \mathrm{E}-04$ & & \\
\hline & PRX & $V^{278} P^{279} L^{280} C^{281} L^{282}$ & $248-328$ & $3.18 \mathrm{E}-02$ & & \\
\hline \multirow[t]{2}{*}{ LILRB3S } & KDO & $W^{54} W^{56} Y^{68} H^{96} V^{97} S^{98} L^{101}$ & $1-120$ & $4.98 \mathrm{E}-08$ & MHC class I, $\beta 2 \mathrm{~m}$ & [33] \\
\hline & IDO & $\mathrm{G}^{145} \mathrm{M}^{146} \mathrm{M}^{217} \mathrm{G}^{218} \mathrm{Q}^{321} \mathrm{~L}^{322}$ & $121-328$ & 8.54E-03 & & \\
\hline
\end{tabular}

LILRB, leukocyte immunoglobulin-like receptors; $M H C$, major histocompatibility complex; ZN, zinc ion; EDO, 1,2-ethanediol; NAD, nicotinamide-adenine-dinucleotide; PRX, adenosine-5'-monophosphate-propyl ester; KDO, 3-Deoxy-D-mano-oct-2-ulosonic; IDO, iodide.

HD11 cell line. Taken together, these data demonstrate that LILRB1 and LILRB3 proteins were constitutively expressed in the transfected HD11 cell line (Supplementary Figure S2B). Moreover, cytotoxicity analysis showed that the proliferation of chicken HD11 cells after transfection was not significantly inhibited or enhanced (Supplementary Figure S2B) and the concentration of NO production was higher in LILRB1- or LILRB3-eGFP group than in the control group (Supplementary Figure S2B). The results indicated that chicken LILRB1 and LILRB3 genes induced the intracellular production of reactive oxygen species in the form of NO.

In humans, some researchers demonstrated that LILRB family genes could bind with MHC class I and $\beta 2 \mathrm{~m}$ [21]. As predicted, GFP-linked LILRB1 and LILRB3 showed a strikingly different pattern of gene expression compared with GFP alone. Our quantitative real-time PCR analysis in cells transfected with GFP-linked LILRB1 and LILRB3 genes and mock control showed that the LILRB1 and LILRB3 genes induced the expression of various members of the MHC class I (HLA-A, $B F-I$, and BF-IV [leukocyte antigens A, avian MHC class I alpha chain 1 and avian MHC class I alpha chain 2]) family as well as other genes involved in class I antigen presentation and processing, such as $\beta 2 m$, transporters associated with antigen processing 1 (TAP1 gene), and TAP2 (Figure 2). The result indicated that upregulation of MHC class I family genes and related genes by LILRB1R and -B3R activation was greater than that by LILRB1S and -B3S (Figure 2). Particularly, the expression of $\beta 2 \mathrm{~m}$ mRNA was markedly upregulated in the HD11 cell line by 191.5-, 125.9-, 96.1-, and 61.2-fold by LILRB1R, -B1S, -B3R and -B3S, respectively. The expression levels of BF-I, $B F-I V, H L A-A$, TAP1, and TAP2 genes were also highly upregulated due to LILRB3R activation by 53.0-, 272.4-, 95.3-, 256.1-, and 144.6-fold, respectively (Figure 2). Moreover, immunoprecipitation analysis demonstrated that LILRB1 and LILRB3 proteins were bound by MHC class I and $\beta 2 \mathrm{~m}$ (Figure 2 ). We confirmed that the LILRB1R, -B1S, -B3R, and -B3S immunoprecipitates contained around $42-$ and $14-\mathrm{kDa}$ size proteins corresponding to anti-MHC class I and $\beta 2 \mathrm{~m}$, respectively
(Figure 2). The amount of LILRB1R, -B1S, -B3R, and -B3S immunoprecipitated with $\mathrm{MHC}$ class I was higher than that with $\beta 2 \mathrm{~m}$. Moreover, the amount of LILRB1R and -B3R precipitated with MHC class I and $\beta 2 \mathrm{~m}$ was higher than LILRB1S and -B3S precipitated with $\mathrm{MHC}$ class I and $\beta 2 \mathrm{~m}$ in macrophage cells, suggesting that the MHC class I and $\beta 2 \mathrm{~m}$ associated with LILRB1R, -B1S, -B3R, and -B3S with different binding affinities (Figure 2).

In addition, flow cytometry analysis using MHC class I- and $\beta 2 \mathrm{~m}$-specific antibodies confirmed an increase in MHC class I surface protein expression in LILRB1 and LILRB3 gene transfected cells. When the expression of LILRB1 and LILRB3 genes was upregulated, MHC class I and functionally related genes were expressed more and this increase in expression was higher in the case of the resistant chicken line 6.3 than in the susceptible chicken line 7.2 (Figure 3). In particular, LILRB1R, -B1S, -B3R and -B3S associated with MHC-I increased by $51.4 \%$, $21.2 \%, 65.6 \%, 59.5 \%$, respectively, and those associated with $\beta 2 \mathrm{~m}$ by $36.5 \%, 34.3 \%, 38.2 \%$, and $33.9 \%$, respectively (Figure 3). In summary, these data indicated that LILRB1 and LILRB3 genes induce the expression of MHC class I and related genes involved in MHC class I antigen presentation and the level of binding with $\mathrm{MHC}$ class I and related genes was higher in line 6.3 than in line 7.2 and differed between the LILRB1 and LILRB3 genes.

\section{SHP-2 associated with phosphorylated LILRB1 and LILRB3 molecules}

The tyrosine phosphatase SHP-2 associates with phosphorylated LILRB groups in B cells, macrophage cells and NK cells [1]. The presence of two ITIMs sequences located within the cytoplasmic region of LILRB1 and LILRB3 genes suggests that they could associate with SHP2 signaling pathways. To determine whether LILRB1 and LILRB3 genes are associated with SHP-2, we examined their tyrosine phosphorylation profiles after transfecting LILRB1-eGFP and LILRB3-eGFP into the HD11 cell line as shown in Figure 4. Firstly, flow cytometry analysis using a p-SHP-2 antibody was performed to confirm 


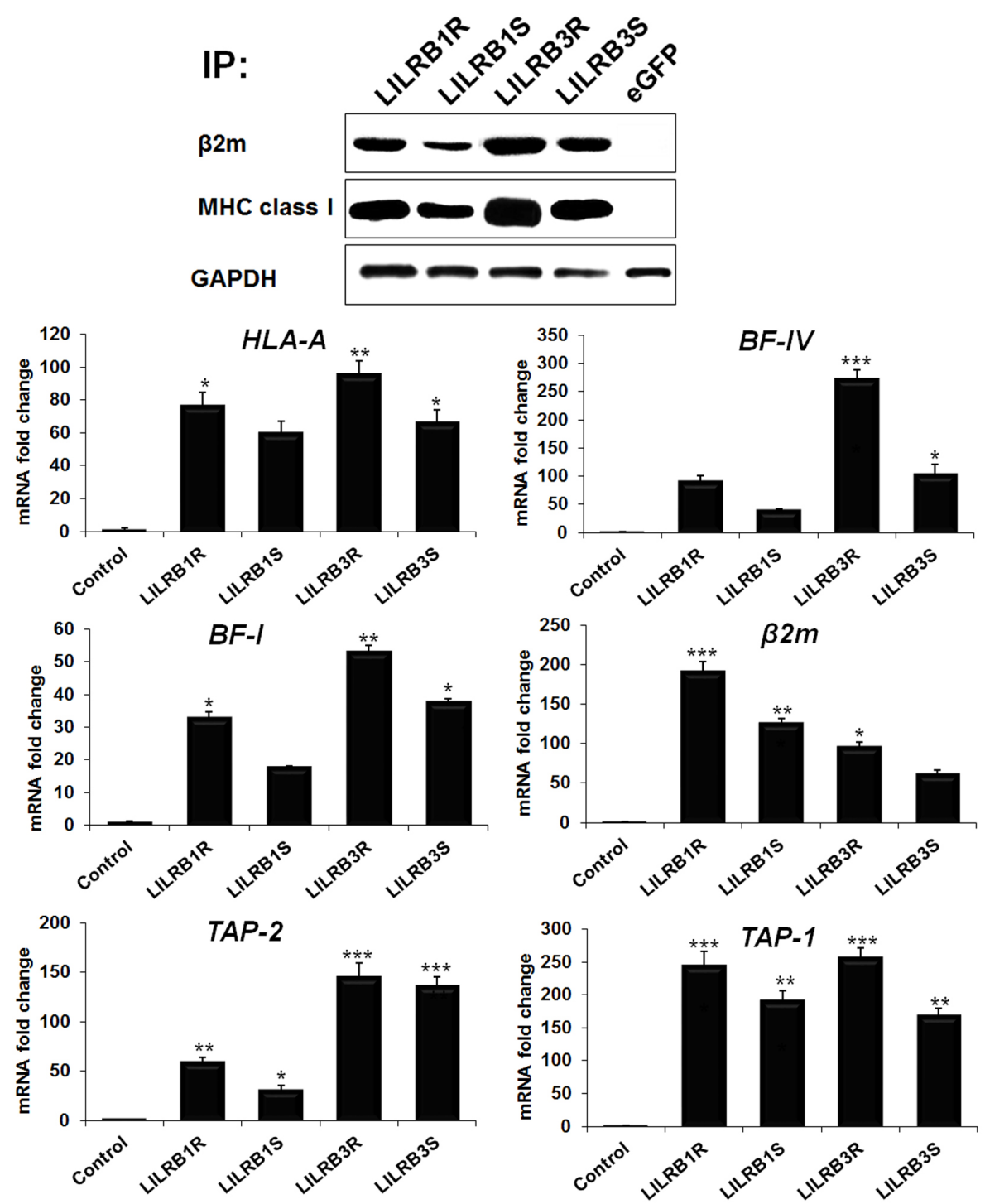

Figure 2. LILRB1 and LILRB3 glycoprotein associated with MHC class I and $\beta 2 \mathrm{~m}$. Top panel, HD 11 cells transfected with LILRB1 and LILRB3 genes from two genetically disparate chicken lines were lysed and then immunoprecipitated with the MHC class I and $\beta 2 \mathrm{~m}$ mAb, then separated by sodium dodecyl sulfate-polyacrylamide gel electrophoresis and immunoblotted with a secondary antibody. Bottom panel, Changes in mRNA levels of the MHC class I family and its related genes in LILRB 1- and LILRB3- transfected cells detected by quantitative reverse-transcription polymerase chain reaction. LILRB, leukocyte immunoglobulin-like receptors; MHC, major histocompatibility complex. Data are presented as the mean \pm standard error of the mean $(n=3)$ of three independent experiments: ${ }^{*} p<0.05$, ${ }^{* *} p<0.01$, and ${ }^{* * *}$ $p<0.001$.

an increase of p-SHP-2 expression in cells transfected with LILRB1 and LILRB3 genes. The expression levels of p-SHP2-associated LILRB1R, -B1S, -B3R and -B3S increased in the macrophage cell line by $20.8 \%, 16.9 \%, 28.7 \%$, and $24.8 \%$, respectively (Figure 4A). It is interesting that the levels of p-SHP2-associated LILRB1 and LILRB3 were higher in line 6.3 than in line 7.2 (Figure 4A). Furthermore, when HD11 cell lysates were immunoprecipitated with anti-GFP $\mathrm{mAb}$ and immunoblotted with an anti-pSHP-2 antibody, approximately $66 \mathrm{kDa}$ size proteins were detectable in the anti-pSHP-2 immunoprecipitates, but not in the mock control. These sizes corresponded to those of LILRB1R, -B1S, -B3R, and -B3S (Figure 4B). These results demonstrate that tyrosine phosphorylation is essential for
SHP-2 binding to LILRB1R, -B1S, -B3R, and -B3S. Moreover, quantitative real-time PCR analysis confirmed the expression levels of SHP-2 in cells transfected with GFP-linked LILRB1, LILRB3 genes and GFP alone (Figure 4B). The SHP-2 mRNA expression was highly upregulated in LILRB3R-transfected HD11 cell line compared to the mock control and was significantly upregulated by 63.7-, 218.7-, 106.2-fold corresponding to LILRB1R, -B3R, and -B3S, respectively (Figure 4B). Thus, we concluded that the expression of SHP-2 in LILRB1R- and -B3R-transfected macrophage cell line was higher than LILRB1S and -B3S-transfected macrophage cell line. Also, LILRB1 and LILRB3 were constitutively tyrosine phosphorylated and associated with SHP-2, which plays an important role in the 

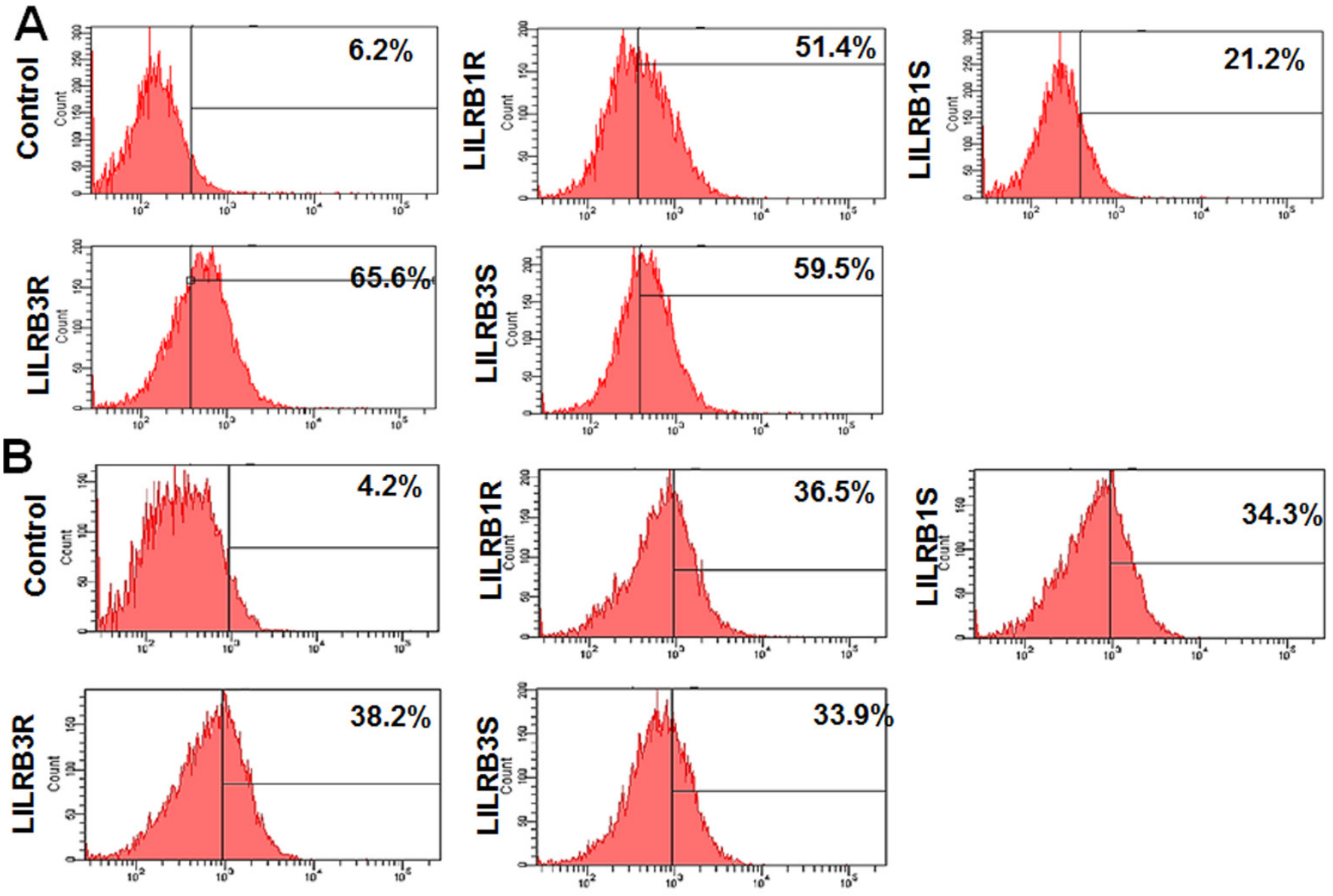

Figure 3. MHC class I and $\beta 2 \mathrm{~m} \mathrm{mAb}$ bind to LILRB1 and LILRB3 gene products from two lines. HD11 cells transfected with eGFP only or eGFP-linked constructs of LILRB1R, -B1S, -B3R, and -B3S stained with MHC class I (A) or $\beta 2 \mathrm{~m}(\mathrm{~B})$ labeled with phycoerythrin. Binding was assessed by fluorescence-activated cell sorting analysis. The resistant (line 6.3) and susceptible (line 7.2) lines are indicated as R and S, respectively. MHC, major histocompatibility complex; LILRB, leukocyte immunoglobulin-like receptors; eGFP, enhanced green fluorescent protein.

modulation of signaling pathways in the chicken macrophage cell line.

LILRB1 and LILRB3 promote the phosphorylation of signaling transduction molecules STAT and JAK The JAK/STAT signaling pathway was investigated to determine the effect of the LILRB1 and LILRB3 genes on signaling pathways. JAK/STAT pathway plays a pivotal role in many important biological responses related to the immune response [22]. Here, we observed that STAT1 $\left(\mathrm{p}-\mathrm{Ser}^{727}\right)$, STAT3 $\left(\mathrm{p}-\mathrm{Ser}^{727}\right)$, and JAK2 ( $\mathrm{p}-\mathrm{Tyr}^{1007} / \mathrm{Tyr}^{1008}$ ) were phosphorylated in LILRB1 and LILRB3 genes-transfected HD11 cell line, while un-phosphorylated (STAT1/3, JAK2, TYK2, and SOCS1) proteins were also detected in LILRB1 and LILRB3 genes-transfected HD11 cell line by western blotting. The level of signaling proteins was higher in LILRB1R- and -B3R- than in -B1S- and -B3S-transfected cell lines (Figure 5A), suggesting that LILRB1 and LILRB3 genes in two genetically disparate chicken lines can differently activate the JAK/STAT signaling pathway.

The transcriptional expression level of the signaling pathway genes was evaluated by qRT-PCR and the results are shown in Figure 5B. The STAT1/3 mRNA expression was highly upregulated in LILRB1R-transfected cells by 205.5 and 103-fold and in LILRB3R-transfected cells by 119.7- and 184.4-fold, respectively. On the other hand, STAT1/3 expression levels of LILRB1S-transfected cells decreased by 82.4-, 55.5-fold and 36.1-, 102.6-fold in LILRB3S-transfected cells, respectively (Figure 5B). Moreover, JAK2 and TYK2 mRNA were more highly expressed in LILRB1R-, LILRB3R-transfected HD11 cells compared to LILRB1S- and LILRB3S-transfected cells. The expression of JAK2 and TYK2 mRNA was markedly upregulated by 63.1-, 286.2-fold, and 29.3-, 128.7- fold in LILRB3Rand -B3S-transfected HD11 cells, respectively (Figure 5B). We also evaluated the expression of SOCS1 by Western blot and qRT-PCR analysis (Figure 5B). SOCS1 protein was strongly detected in LILRB3R gene-transfected cells and qRT-PCR analysis showed strong upregulation of SOCS1 mRNA in LILRB1Rand LILRB3R-transfected cells (Figure 5). In particular, SOCS1 mRNA expression was strongly upregulated by 100.8 -fold in the LILRB3R-transfected HD11 cells (Figure 5B). These results 

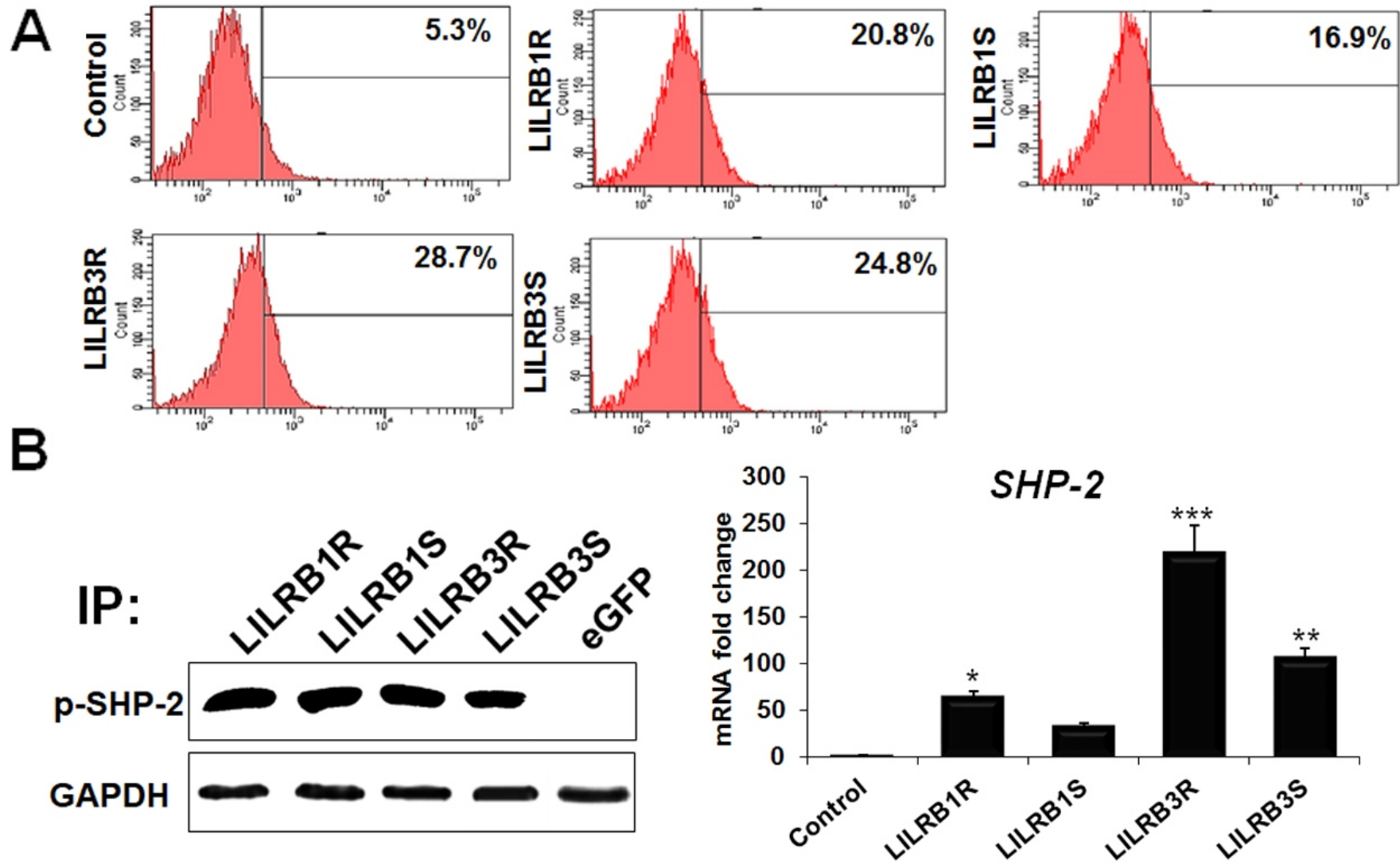

Figure 4. LILRB1R, -B1S, -B3R and -B3S glycoproteins associated with SHP-2. (A) Binding was assessed by fluorescence-activated cell sorting analysis. HD11 cells transfected with eGFP or eGFP-tagged constructs of LILRB1R, -B1S, -B3R, and -B3S stained with SHP-2 mAb and then by Alexa Fluor 488 labeled anti-rabbit secondary antibody. (B) Immunoprecipitation analysis of LILRB1 and LILRB3 associated with SHP-2 (Left) and changes of SHP-2 mRNA levels in cells transfected with LILRB1 and LILRB3 were detected by quantitative reverse-transcription polymerase chain reaction. LILRB, leukocyte immunoglobulin-like receptors; SHP-2, Tyrosine-protein phosphatase non-receptor type 11; HD11, macrophage cells; eGFP, enhanced green fluorescent protein. Data are presented as the mean \pm standard error of the mean $(n=3)$ of three independent experiments: ${ }^{*} p<0.05,{ }^{* *} p<0.01$, and ${ }^{* *} p<0.001$.

indicate that LILRB1 and LILRB3 associate with the JAK/STAT signaling pathway and differentially regulate the immune response depending on the LILRB gene isolated from the two genetically disparate chicken lines.

\section{LILRB 1 and LILRB3 upregulate cytokine genes expression}

To determine the influence of LILRB1 and LILRB3 genes isolated from two chicken lines on the cytokine genes expression in HD11 cell line, qRT-PCR was performed. The expression levels of chemokines, $\mathrm{C}-\mathrm{C}$ motif chemokine ligand (CCL)-4, C-X-C motif chemokine ligand (CXCL)-13, and CXCL-14 mRNA were significantly increased, and particularly they were highly expressed in LILRB3R- transfected cells. Also, the levels of expression of JAK/STAT pathway-associated genes were highly upregulated in LILRB1R, -B3R-transfected cells than in -B1S and -B3S-transfected cells (Figure 6). The Th1-type cytokines, IFN- $\alpha, I F N-\beta, I L-1 \beta$, and IL-6 mRNA were dramatically and differentially upregulated by the LILRB1 and LILRB3 genes transfection. Particularly, expression of IFN- $\alpha$ and IFN- $\beta$ mRNA were increased by $273.4-$ and 213.3 -fold, respectively, in LILRB1R-transfected cells. On the other hand, the expression of $I L-1 \beta$ and $I L-6$ mRNA was upregulated by 123.3- and 149.6-fold, respectively, in LILRB3R-transfected cells (Figure 6). The effects of these transfected genes on the Th2-type cytokines (IL-4 and IL-10), Treg cytokine (transforming growth factor beta $[T G F-\beta] 4$ ), and proinflammatory mediator (lipopolysaccharide-induced tumor necrosis factoralpha factor [LITAF]) were also examined. TGF- $\beta 4$ mRNA was strongly upregulated by 211.5-fold in LILRB1R-transfected cells; and $I L-4, I L-10$, and LITAF mRNA were significantly increased by 138.8-, 151.0-, 178.3-fold, respectively, after transfection with LILRB3R (Figure 6). Moreover, the expression of Th17-type cytokine gene (IL-17F) was also increased by 194.5fold in LILRB3R-transfected cells (Figure 6). Therefore, these results suggest that LILRB1 and LILRB3 genes induce chemokines, Th1/2/17 cytokines production in vitro in activated macrophage cell lines. Also, the expression of genes involved in cytokines production was higher in the resistant chicken line 6.3 than those in the susceptible line 7.2. 

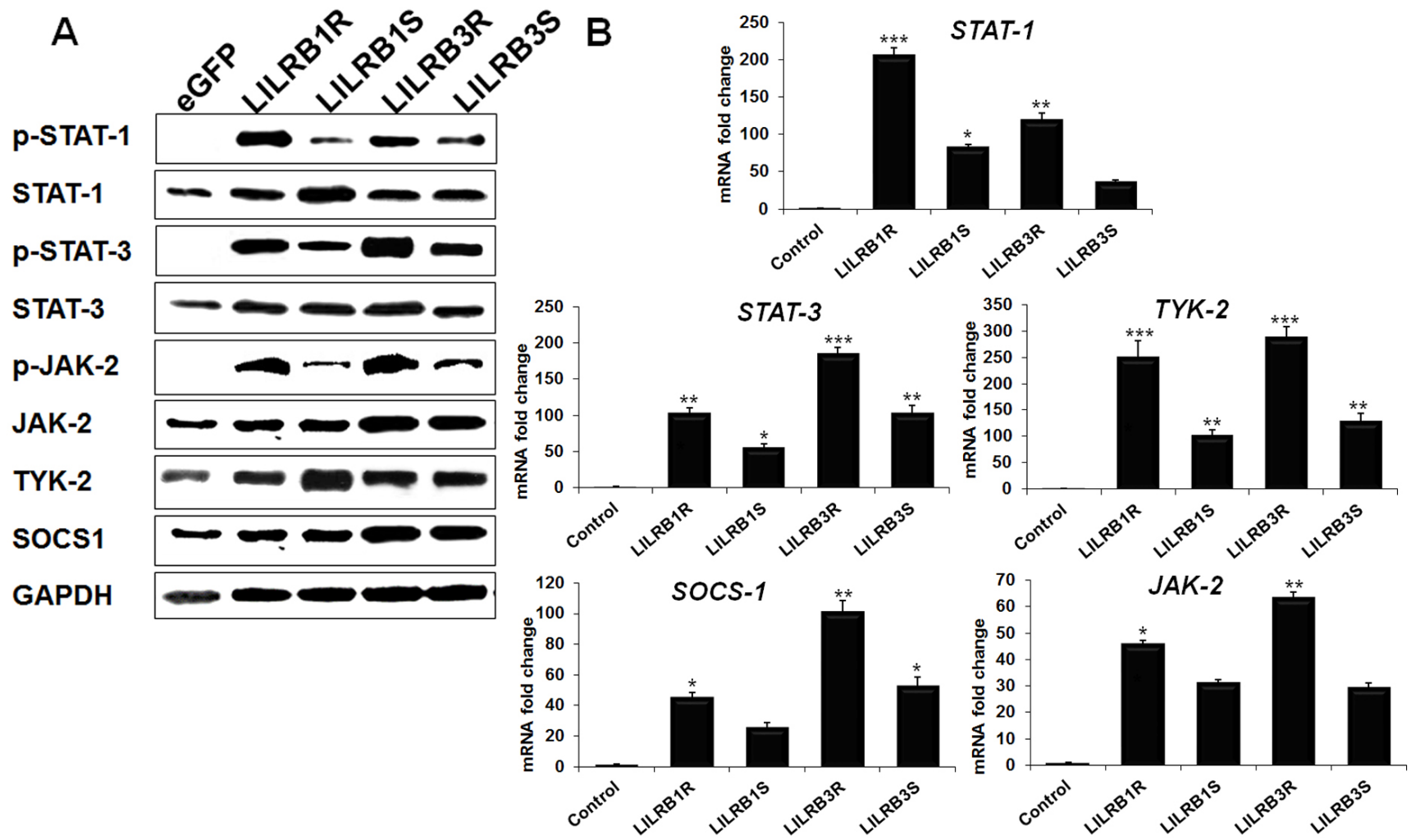

Figure 5. LILRB1R, -B1S, -B3R and -B3S glycoprotein regulated JAK/STAT signaling pathway. (A) Western blotting results of JAK2/TYK2, STAT1/3, and SOCS1 after LILRB1 and LILRB3 transfection in HD11 cell line. (B) Changes in mRNA levels for JAK2/TYK2, STAT1/3, and SOCS1 genes after LILRB1 and LILRB3 gene transfection in HD11 cell line were detected by quantitative reverse-transcription polymerase chain reaction. LILRB, leukocyte immunoglobulin-like receptors; HD11, macrophage cell; JAK2/TYK2, Janus kinase2/tyrosine kinase 2; STAT1/3, signal transducer and activator of transcription1/3; SOCS1, suppressor of cytokine signaling 1. Data are presented as the mean \pm standard error of the mean $(n=3)$ of three independent experiments: ${ }^{*} p<0.05,{ }^{* *} p<0.01$, and ${ }^{* * *} p<0.001$.

LILRB1 and LILRB3 induce cytokines production The expression level of IFN- $\gamma$ mRNA was dramatically increased in the LILRB1R-, -B1S-, -B3R-, and -B3S-transfected HD11 cell lines compared to the mock control by 203.6-, 51.9-, 264.4-, and 157.6-fold, respectively (Figure 7). To determine if the increased IFN- $\gamma$ mRNA expression is mirrored by an increased IFN- $\gamma$ protein in the supernatant, we analyzed the IFN- $\gamma$ protein expression in the supernatant of LILRB1- and LILRB3transfected HD11 cell lines and mock controls by enzymelinked immunosorbent assay. The results revealed significantly higher IFN- $\gamma$ protein levels by $1,691.7,711.1,2,160.2$, and 1,219.82 ng/mL in LILRB1R-, -B1S-, -B3R-, and -B3S-transfected cells lines, compared to the mock control, respectively (Figure 7). The expression level of protein and mRNA transcripts for Th17 cytokine (IL-17A and IL-12p40) showed similar patterns with those of IFN- $\gamma$ (Figure 7). The results indicate that LILRB1R- and LILRB3R-transfected cells produced more IFN- $\gamma$, IL-17A, and IL-12p40 proteins than LILRB1S- and LILRB3S-transfected cells.

\section{DISCUSSION}

In this study, we identified and characterized novel LILRB1 and $B 3$ genes from two genetically disparate chicken lines using a bioinformatics approach and in vitro analysis. Our initial analysis yielded 4 group transcripts as potential LILRB1 and LILRB3 genes from chicken lines 6.3 and 7.2, which were then analyzed using phylogeny alongside other structurally similar Ig-superfamily receptors. Comparison of amino acids identities and similarities between LILRB1 and LILRB3 in line 6.3 to those proteins in line 7.2 ranked between 97\%-99\%, and also between LILRB1 and LILRB3 to the corresponding mammalian proteins ranked between 13\%-19\% and 13\%-69\%, respectively. Moreover, phylogenetic analysis displaying amino acid similarity placed the LILRB1R, -B1S, -B3R, and -B3S together with members of the LILRB1 and LILRB3 genes of mammals suggesting that the 4 receptors are closely associated with LILRB1 and LILRB3 genes from other species. Together with the high degree of sequence similarity, this indicates that the LILRB1R, -B3R, and -B1S, -B3S amino acids isolated from the disease-resistant line 6.3 and susceptible line 7.2 , respectively, represent homologs of the novel receptor in the chicken LILRB family. Moreover, the human LILRB1 and LILRB3 receptors contain 4 canonical and permissive ITIMs with $\mathrm{NxYxxV}$, $\mathrm{VxY} x \mathrm{xV}, \mathrm{VxY} \mathrm{xxL}$ and SxYxxL being present on every receptor [5], whereas the chicken LILRB1R, -B1S, -B3R, and -B3S 

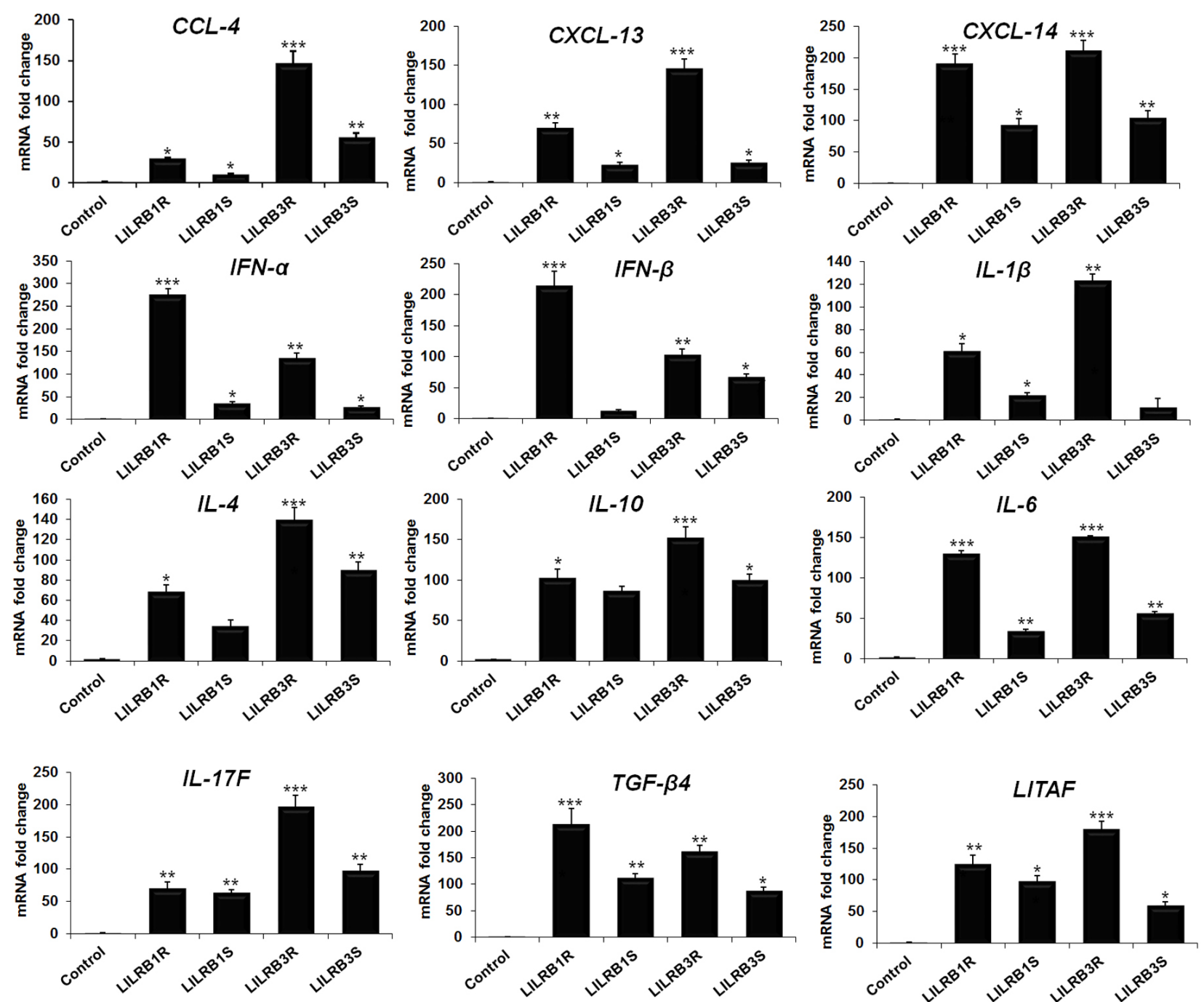

Figure 6. Changes in expression levels of cytokines caused by $L I L R B 1 R,-B 1 S,-B 3 R$, and -B3S genes in transfected HD11 cell line. LILRB, leukocyte immunoglobulin-like receptors; HD11, macrophage cells; CCL, C-C motif chemokine ligand; CXCL, C-X-C motif chemokine ligand; IFN- $\gamma$, interferon- $\gamma$; IL, interleukin; TGF, transforming growth factor beta; LITAF, lipopolysaccharide-induced tumor necrosis factor-alpha factor. Data are presented as the mean \pm standard error of the mean $(n=3)$ of three independent experiments: ${ }^{*} p<0.05,{ }^{* *} p<0.01$, and ${ }^{* * *} p<0.001$.

receptors only contain one canonical ITIMs (LxYxxL) on every receptor. This possibly provides a greater diversity of signaling abilities by human LILRB1 and LILRB3 than that of chicken LILRB1 and LILRB3 genes. Our findings indicate that the LILRB1 and LILRB3 genes can be subdivided into two groups: transmembrane molecules with two ITIM motifs (LxYxxL and $\mathrm{SxVxxV}$ ) in the resistant line 6.3 and two ITIM motifs (LxYxxL and $\mathrm{LxYxxV}$ ) in the susceptible line 7.2. On the other hand, LILRB1R and -B1S have two Ig domains whereas LILRB3R and -B3S contain only one Ig domain (Figure 1C). The results may be implicated in the diversity of signaling abilities for LILRB1 and LILRB3 genes in resistant line 6.3 and susceptible line 7.2. In addition, at least one of these motifs contains the sequence $\mathrm{V} / \mathrm{YxxL} / \mathrm{V}$, which has been shown to be the consensus sequence for binding by the $\mathrm{SH} 2$ domains of the SHP- 1 or SHP-2 phosphatase. Another report had shown that tyrosine- phosphorylated LILRB1 associates and coimmunoprecipitates SHP-1 and SHP-2 [23]. Evidence from other immunoreceptors suggests that the phosphorylation of such motifs generates binding sites for other signaling molecules, including the SHIP [23]. To demonstrate this point, we analyzed the LILRB1 and LILRB3 genes-transfected cell from two chicken lines associated with SHP-2 by immunoprecipitation, FACS analysis, and qRT-PCR. The results indicate that the LILRB1 and LILRB3 genes-transfected cell in the two chicken lines associated with SHP-2 was significantly higher in LILRB3-containing cell line than in LILRB1-containing cell line. In addition, they were higher in the chicken line 6.3 than in line 7.2. Recently, some researchers suggested that SHP-2, a cytoplasmic $\mathrm{SH} 2$ domaincontaining protein tyrosine phosphatase, is involved in the signaling pathways of a variety of growth factors and cytokines such as JAK-STAT and MAPK signaling pathway, and 

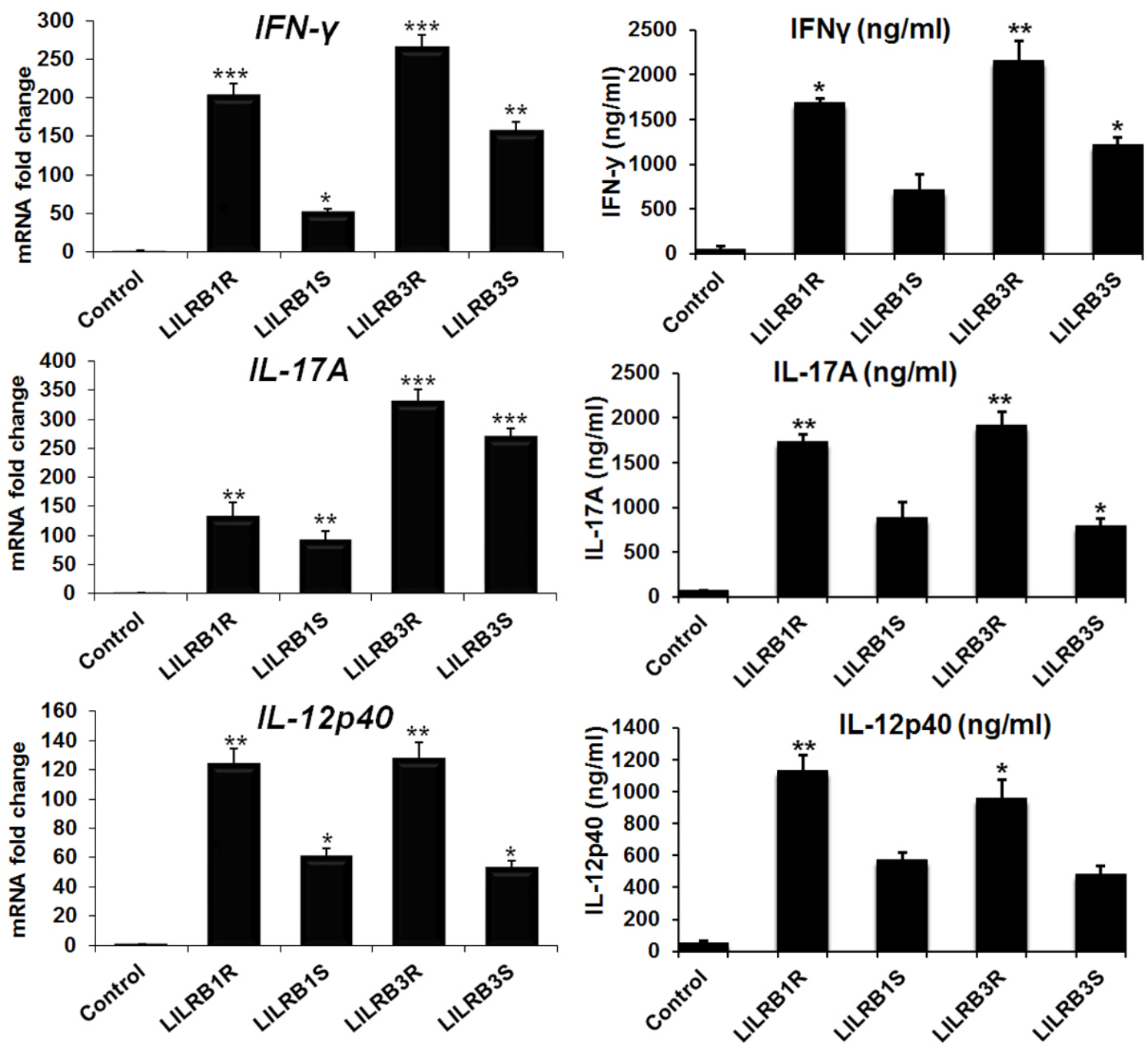

Figure 7. Changes in expression levels of cytokine production caused by LILRB1R, -B1S, -B3R, and -B3S in transfected HD11 cell line. LILRB, leukocyte immunoglobulin-like

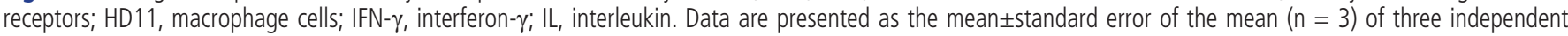
experiments: ${ }^{*} p<0.05$, ${ }^{* *} p<0.01$, and ${ }^{* * *} p<0.001$.

plays an important role in transducing the signal from the cell surface to the nucleus, and is a critical intracellular regulator of cell proliferation and differentiation [4]. Taken together, LILRB1 and LILRB3 genes in the two chicken lines could differentially regulate the signaling pathways and cytokines production.

In humans, the binding of LILRB1, but not LILRB3, to HLA$\mathrm{A},-\mathrm{B}$, and $-\mathrm{C}$, and other non-classical class I molecules such as HLA-E, HLA-G, and HLA-F, is currently being evaluated in several cell types [2]. There are at least two predicted LILRB1 and LILRB3 ligands for each of the LILRB1 and LILRB3 genes, and most of the ligand candidates of these genes bind to MHC class I and $\beta 2 \mathrm{~m}$ antigens. Moreover, these genes are believed to have other ligands or binding residues that differ in the two genetically disparate chicken lines.

By immunoprecipitation, FACS analysis, and qRT-PCR, it was confirmed that LILRB1 and LILRB3 genes in the two chicken lines strongly bind to MHC class I and $\beta 2 \mathrm{~m}$. The LILRB1 and LILRB3 proteins binding to MHC class I was significantly higher than those binding to $\beta 2 \mathrm{~m}$. In addition, the LILRB1 and LILRB3 genes-transfected cell binding to MHC class I and $\beta 2 \mathrm{~m}$ in line 6.3 were markedly higher than those in line 7.2. Moreover, LILRB1 and LILRB3 genes significantly upregulated MHC class I-related genes such as TAP-1 and TAP-2, which are essential for antigen presentation by the MHC class I pathway, by more than 100 -fold. The finding that MHC class I is recognized by inhibitory receptor genes LILRB1 and LILRB3 in two genetically disparate chicken lines expressed on macrophage cells expands the concept of self-recognition in immune response. Taken together, our results show that LILRB1 and LILRB3 genes are necessary and sufficient for the induction of MHC class I expression. Future analyses of the in vivo function of LILRB1 and LILRB3 genes are required to reveal if these two molecules play redundant or more exclusive roles in MHC class I-dependent immune responses. 
A deeper understanding of the intracellular signal transduction pathways initiated by LILRB1 and LILRB3 genes is necessary to know how these genes can activate the expression of immune-related genes. Concurrent with a previous report showing that JAK/STAT pathway plays a key role in cytokineinduced biological responses, we demonstrated the effects of LILRB1 and LILRB3 genes on the JAK/STAT signaling pathway in the activated HD11 cell line [24]. Moreover, LILRB groups have been shown to regulate TLR activity [8], antigen-presenting phenotype and cytokine production [8]. Thus, the LILRB groups play an important role in the regulation of innate immune responses. The expression levels of phosphorylated STAT1 $\left(\mathrm{p}-\mathrm{Ser}^{727}\right)$, STAT3 $\left(\mathrm{p}-S e r^{727}\right)$, and JAK2 (p-Tyr $\left.{ }^{1007} / \mathrm{Tyr}^{1008}\right)$ molecules and un-phosphorylated (STAT1/3, JAK2, TYK2, and SOCS1) molecules in response to LILRB1 and LILRB3 genetransfected HD11 cell line compared to mock control by western blotting were increased, and also significantly upregulated in STAT1/3, JAK2, TYK2, and SOCS1 mRNA by qRT-PCR. Our results indicate that LILRB1 and LILRB3 activate and regulate the JAK/STAT signaling pathway and also control the immune system.

This study provides the first evidence indicating the possible role of JAK/STAT pathway in the signaling mechanism of chicken LILRB1 and LILRB3 genes in the chicken HD11 cell line. To investigate the function of LILRB1 and LILRB3 genes for growth factor activation, 3 chemokine (CCL-4, CXCL13 , and $C X C L-14)$ and 12 cytokine (IL-1 $\beta, I L-4, I L-6, I L-10$, $I L-12 p 40, I L-17 A, I L-17 F, I F N-\alpha, I F N-\beta, I F N-\gamma, L I T A F$, and TGF $\beta 4$ ) genes in the immune cell were measured by qRT-PCR. The expression of these cytokine genes was increased in LILRB1 and LILRB3-transfected cells of line 6.3 than those of line 7.2. As previously reported, chemokines appear to promote host resistance by mobilizing leukocytes and activating immune functions that kill, expel, or sequester pathogens [25] and IFN- $\alpha$, IFN- $\beta$, IFN- $\gamma$, and IL- 17 families play important roles in conferring resistance against pathogens. They also increase the expression of class I MHC molecules on cells making them more resistant to pathogens and are highly produced from Th1 and Th17 cells in response to pathogens [26]. IFN and IL-17 induce IL-1 $\beta$, IL-6, LITAF [27] and TGF $\beta 4$ mRNA production [26]. Moreover, Th2 cytokine (IL-10) and Treg cytokine (TGF 34 ) mRNA have been reported to upregulate the cell surface expression of the inhibitory receptors on monocytes and dendritic cells and these mRNA also associated with the LILRB group in response to pathogens such as HIV [28]. Our result indicates that chicken LILRB1 and LILRB3 genes activate the JAK/STAT signaling pathway, and upregulate the expression of chemokines such as Th1, Th2, and Th17 cytokines. Expression levels of chemokines and cytokines were significantly higher in LILRB1- and LILRB3-transfected cells of the resistant line 6.3 than those of the susceptible line 7.2. Therefore, we demonstrated that LILRB1 and LILRB3 genes can induce several chemokines, such as Th1, Th2, and Th17 cytokine mRNA, which may be essential in the pathogenesis of diseases, highlighting the importance of this novel pathway in chicken disease.

In summary, this is the first report of the cloning, structural analysis, and function of the novel chicken LILRB1 and LILRB3 genes isolated from two genetically disparate chicken lines. Like other LILRB family members, LILRB1 and LILRB3 genes possess ITIM motifs in the cytoplasmic domain that bind to cytosolic tyrosine kinases such as SHP-2. We also showed that the LILRB1 and LILRB3 genes bind to MHC class I and $\beta 2 \mathrm{~m}$ as well as other genes involved in class I antigen presentation, processing and regulation of immune responses. Moreover, LILRB1 and LILRB3 genes induce and regulate the JAK-STAT signaling pathway and upregulate Th1, Th2, and Th17 cytokine genes in the chicken cell line.

\section{CONFLICT OF INTEREST}

We certify that there is no conflict of interest with any financial organization regarding the material discussed in the manuscript.

\section{ACKNOWLEDGMENTS}

This work was supported by the Chung-Ang University Research Scholarship Grants in 2017 and NIFA grant (\#20176701526793) of USDA.

\section{REFERENCES}

1. Kang X, Kim J, Deng M, et al. Inhibitory leukocyte immunoglobulin-like receptors: immune checkpoint proteins and tumor sustaining factors. Cell Cycle 2016;15:25-40.

2. Zhang Z, Hatano H, Shaw J, et al. The Leukocyte immunoglobulin-like receptor family member LILRB5 binds to HLA-class I heavy chains. PLoS One 2015;10:e0129063.

3. Borges L, Hsu ML, Fanger N, Kubin M, Cosman D. A family of human lymphoid and myeloid Ig-like receptors, some of which bind to MHC class I molecules. J Immunol 1997;159: 5192-6.

4. Burshtyn DN, Yang W, Yi T, Long EO. A novel phosphotyrosine motif with a critical amino acid at position -2 for the $\mathrm{SH} 2$ domain-mediated activation of the tyrosine phosphatase SHP-1. J Biol Chem 1997;272:13066-72.

5. Vivier E, Daeron M. Immunoreceptor tyrosine-based inhibition motifs. Immunol Today 1997;18:286-91.

6. Zhang F, Zheng J, Kang X, et al. Inhibitory leukocyte immunoglobulin-like receptors in cancer development. Sci China Life Sci 2015;58:1216-25.

7. Billadeau DD, Leibson PJ. ITAMs versus ITIMs: striking a balance during cell regulation. J Clin Invest 2002;109:161-8. 
8. Brown DP, Jones DC, Anderson KJ, et al. The inhibitory receptor LILRB4 (ILT3) modulates antigen presenting cell phenotype and, along with LILRB2 (ILT4), is upregulated in response to Salmonella infection. BMC Immunol 2009;10:56.

9. Rothe K, Quandt D, Schubert K, et al. Latent cytomegalovirus infection in rheumatoid arthritis and increased frequencies of cytolytic LIR-1+CD8+ T cells. Arthritis Rheumatol 2016;68: 337-46.

10. Hogan LE, Jones DC, Allen RL. Expression of the innate immune receptor LILRB5 on monocytes is associated with mycobacteria exposure. Sci Rep 2016;6:21780.

11. Tedla N, An H, Borges L, et al. Expression of activating and inhibitory leukocyte immunoglobulin-like receptors in rheumatoid synovium: correlations to disease activity. Tissue Antigens 2011; 77:305-16.

12. Kim T, Vidal GS, Djurisic M, et al. Human LilrB2 is a betaamyloid receptor and its murine homolog PirB regulates synaptic plasticity in an Alzheimer's model. Science 2013;341:1399404.

13. Bergamini A, Chimenti MS, Baffari E, et al. Downregulation of immunoglobulin-like transcript-4 (ILT4) in patients with psoriatic arthritis. PLoS One 2014;9:e92018.

14. Bashirova AA, Martin-Gayo E, Jones DC, et al. LILRB2 interaction with HLA class I correlates with control of HIV-1 infection. PLoS Genet 2014;10:e1004196.

15. Jansen CA, van Haarlem DA, Sperling B, et al. Identification of an activating chicken ig-like receptor recognizing avian influenza viruses. J Immunol 2016;197:4696-703.

16. Truong AD, Hong YH, Lillehoj HS. High-throughput sequencing reveals differing immune responses in the intestinal mucosa of two inbred lines afflicted with necrotic enteritis. Vet Immunol Immunopathol 2015;166:116-24.

17. Truong AD, Hong YH, Lillehoj HS. RNA-seq profiles of immune related genes in the spleen of necrotic enteritis-afflicted chicken lines. Asian-Australas J Anim Sci 2015;28:1496-511.

18. Burge CB, Karlin S. Finding the genes in genomic DNA. Curr Opin Struct Biol 1998;8:346-54.

19. Klasing KC, Peng RK. Influence of cell sources, stimulating agents, and incubation conditions on release of interleukin-1 from chicken macrophages. Dev Comp Immunol 1987;11:38594.

20. Truong AD, Hong Y, Hoang CT, Lee J, Hong YH. Chicken IL-26 regulates immune responses through the JAK/STAT and NFkappaB signaling pathways. Dev Comp Immunol 2017;73:1020.

21. Takahashi A, Kuroki K, Okabe Y, et al. The immunosuppressive effect of domain-deleted dimer of HLA-G2 isoform in collagen- induced arthritis mice. Hum Immunol 2016;77:754-9.

22. Truong AD, Rengaraj D, Hong Y, et al. Differentially expressed JAK-STAT signaling pathway genes and target microRNAs in the spleen of necrotic enteritis-afflicted chicken lines. Res Vet Sci 2017;115:235-43.

23. Colonna M, Samaridis J, Cella M, et al. Human myelomonocytic cells express an inhibitory receptor for classical and nonclassical MHC class I molecules. J Immunol 1998;160:3096100 .

24. Truong AD, Rengaraj D, Hong Y, et al. Analysis of JAK-STAT signaling pathway genes and their microRNAs in the intestinal mucosa of genetically disparate chicken lines induced with necrotic enteritis. Vet Immunol Immunopathol 2017;187:1-9.

25. Chensue SW. Molecular machinations: chemokine signals in host-pathogen interactions. Clin Microbiol Rev 2001;14:82135.

26. Vilcek J, Feldmann M. Historical review: cytokines as therapeutics and targets of therapeutics. Trends Pharmacol Sci 2004;25: 201-9.

27.Hong YH, Lillehoj HS, Lee SH, Park D, Lillehoj EP. Molecular cloning and characterization of chicken lipopolysaccharideinduced TNF-alpha factor (LITAF). Dev Comp Immunol 2006; 30:919-29.

28. Beinhauer BG, McBride JM, Graf P, et al. Interleukin 10 regulates cell surface and soluble LIR-2 (CD85d) expression on dendritic cells resulting in T cell hyporesponsiveness in vitro. Eur J Immunol 2004;34:74-80.

29. Gomez-Lozano N, Estefania E, Williams F, et al. The silent KIR3DP1 gene (CD158c) is transcribed and might encode a secreted receptor in a minority of humans, in whom the KIR3DP1, KIR2DL4 and KIR3DL1/KIR3DS1 genes are duplicated. Eur J Immunol 2005;35:16-24.

30.Sliz P, Michielin O, Cerottini JC, et al. Crystal structures of two closely related but antigenically distinct HLA-A2/melanocytemelanoma tumor-antigen peptide complexes. J Immunol 2001; 167:3276-84.

31. Tormo J, Natarajan K, Margulies DH, Mariuzza RA. Crystal structure of a lectin-like natural killer cell receptor bound to its MHC class I ligand. Nature 1999;402:623-31.

32.Schiefner A, Fujio M, Wu D, Wong CH, Wilson IA. Structural evaluation of potent NKT cell agonists: implications for design of novel stimulatory ligands. J Mol Biol 2009;394:71-82.

33. Brooks CL, Muller-Loennies S, Brade L, et al. Exploration of specificity in germline monoclonal antibody recognition of a range of natural and synthetic epitopes. J Mol Biol 2008;377: 450-68. 